\title{
Potential barriers in the therapeutic relationship in unaccompanied refugee minors in mental health
}

\begin{abstract}
Background: Unaccompanied refugee minors present with disproportionately high prevalence of emotional and psychological morbidities. However, their utilization of mental health services has been shown to be significantly poorer than the indigenous population of the country they seek asylum in. Despite this, there is limited research exploring their perspectives on the barriers they face.
\end{abstract}

Aims: This research aims to understand unaccompanied refugee minors' and their carers' perceptions of the barriers to utilize mental health services and to explore issues perceived to be related to poor engagement with services.

Methods: Semi-structured interviews with fifteen unaccompanied refugee minors and their carers were conducted. This was to elicit their views, perceptions and beliefs based on their experience of receiving treatment from a specialist mental health service in the UK.

Results: Thematic analysis was used and the findings were categorised into two broad themes; the participants' perceptions of the intervention and perceptions of the professionals. Salient aspects of these are discussed.

Conclusions: The findings have potential to stimulate further research into gaining a better understanding of the barriers these young people face in accessing help, and may contribute to developing services that are more efficient in engaging this vulnerable group and meeting their specific needs. 


\section{Introduction}

Developing appropriate mental health support for unaccompanied refugee minors is essential for effective clinical care and it cannot be assumed that existing services are meeting this need. Recent estimates illustrated that globally there are approximately 12 million refugees and asylum-seekers (UNHCR, 2012) and a growing crisis is the number of young people displaced from their home countries. This is reflected in work that shows approximately half of the world's refugees are adolescents, many being unaccompanied, as they are under 18 years and separated from parents (Thomas \& Byford, 2003).

Refugees are a high-risk group for developing mental health difficulties (Fazel et al., 2005) as their exposure to adversity and trauma is associated with a range of negative psychological outcomes (Fazel et al., 2012; Thabet et al., 2006). For example, high numbers experience severe symptoms of anxiety, depression and posttraumatic stress (Derluyn \& Broekaert, 2007). Consequently, they often require mental health services (Huemer \& Vostanis, 2010), but typically struggle to get their mental health needs met (Ellis et al., 2011). This group tend to be reluctant to access services and often fail to engage in treatment (Sanchez-Cao et al., 2012). Language barriers; lack of knowledge about services; high frequency of relocation; and varied cultural understandings of psychopathology and treatment have been suggested as underlying influencing factors (Sanchez-Cao et al., 2012). Engagement strategies have included outreach models (Harris, 2008), cultural training for professionals (Ehntholt \& Yule, 2006), quick access to services (Kennedy et al., 1999), and school-based mental health interventions (Kia-Keeting \& Ellis, 2007; Chiumento et al., 2011), yet there is a paucity of evidence on service utilization patterns in this population. 
It cannot always be assumed that stressful past experiences render refugee minors as psychologically vulnerable or that they need therapy (Summerfield, 2000). Nonetheless, what is evident is that the assessment and mental health care of refugee minors involves special knowledge and skills. Planning interventions must therefore account for their specific needs (Westermeyer, 1991) as there is potential for failure to consider the distress experienced and adversities encountered (Ehnhold \& Yule, 2006). Problematically, the evidence-base on efficacy of specific treatment modalities remains scarce (Huemer \& Vostanis, 2010). It is clear, therefore, that it is essential to have a better understanding of the perspectives of unaccompanied refugee minors, particularly about how services might be tailored to meet their needs (Rutter, 2003).

\section{Aims of the paper}

The purpose of this paper is to examine mental health services from the perspective of unaccompanied refugee minors and their carers. Specifically, this research is expected to contribute to an understanding of the possible barriers to engagement and to identify aspects of service provision that contribute to the observed poor service access and treatment engagement. The research question addressed is 'what are the perceptions of unaccompanied refugee minors of their treatment and practitioner and what does that teach us about engagement?'

\section{Method}

\section{Sample}


Unaccompanied refugee minors aged 15-18 years (table 1) and their carers (table 2) were recruited through two different Local Authorities in England. There were 15 minors, mostly from Afghanistan, Somalia and Iran, presenting predominantly with PTSD, depression and self-harm involved with Child and Adolescent Mental Health Services (CAMHS), and 15 carers (predominantly foster carers, but in some cases residential care home staff).

INSERT TABLES HERE

\section{Data collection}

Semi-structured interviews were used to explore the young persons' and carers' perspectives, as including young people in mental health research ensures that children's voices influence the development of services (see Mawn et al., 2015). Interviewing continued until thematic saturation was reached, noted by the repetition of issues (O’Reilly \& Parker, 2013) and following the criteria laid out by Francis et al. (2010).

\section{Analysis}

Thematic analysis was utilised due to its efficiency in drawing underlying meanings and participant perspectives (Braun \& Clarke, 2006). This was appropriate for an exploratory study as there is limited work examining the perspectives of unaccompanied refugee minors with mental health difficulties. NVivo software was used to facilitate coding.

\section{Ethics}


Ethical approval was provided by the UK National Research Ethics Service, following rigorous consideration of the specific ethical issues related to this group. Informed consent was taken from all parties and capacity was judged by the mental health practitioner working with the young people.

\section{Results}

The data revealed two core themes. First, young people provided perspectives of the practitioners with whom they engaged and, second, they revealed perceptions of the therapeutic practices utilised.

\section{Theme one: Young people's perceptions of their practitioner}

Young people had specific views of the practitioner working with them, and they talked about the preconceived ideas they held of the professionals involved in their care, which were influenced by their views of gender and ethnicity. Their views of services were informed by their interaction with the mental health practitioner and was generally positive; however, they described potential barriers to this process.

The young people perceived the relationship asymmetrical, positioning themselves as 'patient' and the practitioner 'doctor' as an authority figure holding primary epistemic rights. In turn, young people felt their role was to follow instruction. 
1. Interviewer-“I mean, if it is not helping, why do you still take?” YP-“Uh, because she [psychiatrist] says to......”

2. "You know, like when I have some question, I say to the doctor just like this, he give me reasons about that, and then I think maybe the doctor is right."

3. "I say, okay, I deal with this, okay, (but) you know more than me, so I will go ahead with it."

Demonstrated by the young people here is a more passive position of following direction of the professional; 'because she says to', 'I think maybe the doctor is right', and 'you know more than me so I will go ahead'. The specific characteristics of the practitioner was perceived as being influential for the relationship between the young person and the mental health practitioner. The presence or absence certain characteristics of practitioners appeared important for engagement, especially the ability of the young people to view them as individuals with positive attributes.

4. "if they have that sense of humour, it's perfect, it's fine, so you have a laugh you know." 
5. “What I liked some people like nice personality, like you and A [initial of clinician], anyone, speak to me. Is like lovely personality."

Nonetheless, despite this generally positive appraisal of the personal characteristics of the practitioner, when considering the role played in their care, such descriptions were more problematic. Indeed, the way they were treated by the practitioner was of central importance to the young people;

6. “Talk to me as me, I'm not like baby, man.”

7. "I'm just (made) a mistake, see, it's my mum this picture and nobody explain to me, police don't understand me. I'm not British, I'm from Africa and people don't even understand, I didn't speak English that time and nobody bring an interpreter."

8. "I was thinking at that time that the person is over-confident that he has diagnosed $S$ (young person) very quickly. And they said ah well I know, I've dealt with a lot of people, this, this, this. I think that was not professional.”

In general, some participants held quite negative views of the practitioners. They felt that the process infantilises them 'I'm not like baby' and felt patronised. Also, the need to be understood in their own languages was an essential part of their identity with the perception 
that their treatment had been in error due to this misunderstanding. Additional to these perceptions, there were also specifics that appeared to affect the therapeutic dynamic. For example, carers commented that engagement with the young people seemed to be affected by the gender of practitioners. Talking about emotions could be difficult with women and this appeared to relate to the perception that women needed to be protected and prevented from experiencing any distress.

9. "I think for somebody whose whole world is totally different, and especially when you've got to talk and you're talking to a woman as well, in there, you know, sitting in a room talking to a woman about your inner feelings or whatever, I think they weren't comfortable about that."

10. "To S [young person], women need to be protected, women need to just see the soft side of things and don't have to suffer, and if there is any burden of suffering, it is good for the man to carry this burden and not for a woman.....”

The belief that "women need to just see the soft side of things" may have prevented some participants from discussing their emotions and anything they deemed traumatic with female therapists. The perception of their masculinity of "good for the man to carry this burden" further reinforced this gender difference. Therefore, the practitioner's gender can have a significant effect on the therapeutic interaction and needs to be accounted for. 
Equally, the ethnicity of the practitioner was reported as important, and positioned as a potential barrier for service engagement.

11. "English people and doctor people, you don't understand me anything at all for me."

12. "I trust hundred per cent, ninety, ninety-five per cent English people, but Asian people, other Asian people tell you wrong way in United Kingdom.”

Generally, the young people felt that practitioners were not able to understand their needs. Although some did trust English practitioners, people of other cultures were viewed with some suspicion, for example, young person 9 'Asian people tell you the wrong way'. Carers also recognised that culture had a significant effect on helping and identified that they would relate better to those who had a similar background, possibly from their own community. Even outside their community, as one carer reflected, young people may relate better with practitioners of the same skin colour.

13. "I think if there was a culture, uh, common thing within (between) a young person and the person who's working, I think that generally works.”

14. "To him, because of the colour of skin, I'm the same colour of skin, it made him feel at ease, definitely.” 
However, some carers felt that this separation of the young people was detrimental and implied that integration would be a better way forward 'we should be helping mix'.

15. "You don't help them if you keep them aside, if you keep them in their own groups, you're not helping them, you're creating classes among classes and among classes. I think this is a wrong policy, and I think your services shouldn't be, you know, we should be helping mix"

Carer 10

The characteristics of the individual practitioner appear to have either a positive or negative effect on the therapeutic interaction. While some attributes, such as sense of humour and personality, may be adjustable in dealing with this client group, but other characteristics such as gender and ethnic background are potentially fundamental barriers, which are much more difficult to address.

\section{Theme two: Young people's perceptions of therapies}

While the young people saw the role of the practitioner as central to their engagement with services, the nature of the therapies itself was also important. Participants discussed their personal experiences of interventions received. Typically, these young people were engaged with a range of different treatments at the time of being interviewed, which included psychological, activity-based and pharmacological. Contemporary practice in mental health uses several talking therapies and this was commented on by the participants. Mostly the young people did not recognise the benefits from talking therapies and their experiences 
tended to range from passively following instructions (as we noted earlier) to actively finding them unpleasant or unnecessary.

16. “...they keep talking to you, that means this person annoying you and you just closing your ears.”

17. “At first he didn't know what this therapy was. He kept saying, why are they asking me all this, he didn't realise talking about a problem even helped him. He didn't know that."

Carer 3

When finding talking uncomfortable, young people could disengage from the therapeutic process 'you just close your ears', which obviously has implications for outcomes.

Additionally, the slow pace of therapy, together with the need to readdress issues from their past were also viewed negatively. The misunderstanding of the need to do this in therapy may reflect language or culture, but is potentially a barrier to engagement.

18. "Every time when I come it's like a rotation or same thing."

19. "So when you're talking, it's too slow for them, you know, it's too slow, so they don't want to engage in it, 'cos they're not seeing any immediate (benefit)." 
Sometimes talking was even perceived to make their presentation worse by reminding them of traumatic memories that they thought they had put behind them. The intervention reawakened memories of their experiences in their country of origin and relocation to their host country that they thought had led to the current mental health concerns. This was mostly perceived by the young people as regressing rather than making progress.

20. "So, when I went back home, I was thinking about that, especially that time I was talking with the doctors, so I was thinking about him, home, what happened why this happened to me, why I been in this problem and why I've been faced with this, uh, depression, that stuff. So it's quite, wasn't helping me the way talks.”

21. “...... talks doesn't helps me because every time I was talking about it, it just reminded me about home and was hurting me about same, same problem and more worse."

Compared to talking therapy, medication seemed to generate more positive views of treatment; in helping them to feel better, lifting their mood, stopping their thinking, or helping them forget.

22. "That medicine I was taking, it was anti-depressious, so I was keeping my mood up, so quite was that bit helping me when I was feeling down."

23. “...... if you give me tablet I might get better, that's why I want to see P (CAMHS psychiatrist)." 
The basis of these beliefs may be the young persons' experience of medical treatments for physical conditions and a belief in the power of medication for mental health difficulties. It appears that, for these young people, suppressing their traumatic past experiences and concentrating on solving future problems was the best way of coping. This also helped them avoid being overwhelmed by a combination of distressing past events and an uncertain future. However, talking therapy was largely perceived as a negative process as it encouraged them to explore and process these memories, rather than repress them. Some of those interviewed, however, did find the experience of talking helpful. One young person, reported that therapy enabled him to talk about his problems, and another found the experience of sharing their stories cathartic; especially as the clinician was impartial and not related to them in their personal life.

24. "She talked to me about it, and I think it was kind of helpful, because sometimes when you talk to people, you know, everything's coming out...... and you feel a bit better. 'Cos if you keep inside for a long time.”

The perception of these young people was that their culture does not encourage them to talk about difficult personal and social issues due to associated shame and guilt. Carers reflected that these young people need a lot of reassurance about the therapist's neutrality before engaging. Moreover, the therapist needs to demonstrate an explicit interest, manifested by active listening and offering practical help. 
25. “ especially the society where we come from, children don't talk a lot. Children are not even allowed to reveal things. Children are not even allowed to talk about being abused."

Carer 4

They particularly found it very upsetting to talk about the family they have been separated from at a young age. Carers reiterated this view by adding that the young people actively resisted remembering past life events, as they were too painful. When this was attempted by the mental health professionals, the young people struggled, and this could even lead to worsening of their distress and symptoms.

26. "Uh, that doesn't helps me, that makes me more hard because, um, the all the time I was talking about the past, so every time I went there reminding me after I went home again, same depression and same problems."

27. "Some of them is talking about your family, 'cos I don't want to talk about my family, 'cos I was, I was young when I left them. When I talk about, I feel really bad, just feel really sad, crying."

Nevertheless, re-processing traumatic memories has been shown to be necessary in treating mental health problems (Bevin, 1999), even if the young people do not always appreciate the relevance, at least at the outset. In this study, participants wanted to focus on solving the current and future problems instead. Participants expected d discussions on current problems 
rather than the past, as well as practical tips and strategies to address those. They wanted the mental health practitioners to talk about their immigration problems, education, substance misuse, sleep difficulties and other current difficulties.

28. “...... not even talk about what happened but talk about what's happening to him now, like his sleep problems and some of his symptoms."

Carer 13

\section{Discussion}

Unaccompanied refugee minors provide a unique challenge to current available services. The participants expressed negative and positive views of their mental health care, and this appeared influenced at times by a range of diverse factors. While some young people and their carers appreciated the help provided, they would often represent themselves as passively engaged participants in treatment. This may reflect their perception of a clear epistemic hierarchy of authority, which may be secondary to their age, culture, or uncertainty around their refugee status. However, certain characteristics of the practitioners appeared important, such as humour and 'nice' personality. While it would be expected that practitioners have an engaging manner, characteristics such as gender and ethnicity, which some young people struggled with, would be harder to overcome. Additionally, some young people found talk as their modality of change difficult to comprehend; especially, the need to revisit difficult memories as part of the therapeutic process. This appeared to be complicated around cultural beliefs around expressing emotions openly, but also the belief that medication would be preferable and sufficient. 
Developing effective therapeutic services for children can be complicated as the tendency of service development is to focus on the modality of the therapy as the essential component of care. Influences such as the available evidence base or pre-existing models may dominate, but other factors such as developing an early therapeutic alliance and collaboration with the practitioner are probably more important. While the characteristics of the practitioner may be important to most children and families, the current socio-political environment with its emphasis on equality and diversity may not allow this to be freely expressed. These young people however, have been able to clearly outline the factors that they feel influences their therapeutic engagement. Although engagement of the practitioner should be a paramount concern, this needs to be balanced with the contemporary ethical environment. Thomas (2007) proposed that practitioners from the refugee's own ethnic communities may be more desirable by the service users, but this may not be possible practically or ethically. Furthermore, it could be viewed as discriminatory to only allocate young people to practitioners based on gender or ethnicity (Legislation.gov.uk, 2010). Therefore, practitioners need to be aware of this dynamic in forming the relationship with the young person and addressing it if necessary. This is particularly important as practitioners need to be competent to communicate with their patient in ways that facilitate good therapeutic relationships to achieve clinical aims (Priebe \& McCabe, 2008).

While it is clear there are additional complications in establishing a therapeutic relationship with unaccompanied refugee minors, it would be problematic for them to express this concern if they view an asymmetrical power relationship with the practitioner. Despite the current discourse on child-centred practice, which places the child at the centre of decisionmaking (Söderbäck, 2011), the relationship is unlikely to be fully equal. Additionally, the asymmetrical relationship perceived by the young person, with the professional dictating the 
relationship may influence the direction of therapy. The expectation of Western therapies is to allow the young person to reach the desired outcome through reflection on their problems, which again is underpinned by child-centred practice. The unaccompanied refugee minor may, however, find this uncomfortable if it does not mirror their underlying cultural beliefs. Although this may not immediately be a barrier in therapy, it may result in poorer longerterm outcomes.

Therapy itself may have some intrinsic qualities that impact on effectiveness. The conceptualisation of mental health in the West has been heavily informed by medical ideas that elsewhere may be viewed as having a social or spiritual emphasis (Honwana, 2006; Lustig et al., 2004). Therefore, treatments that have evolved rely on verbal dialogue between practitioner and child, but this may not be as effective with children from other cultures (Lustig et al., 2004). This view may be further influenced from the culturally informed inhibition in talking about one's private life, especially adverse experiences, and the cultural attitude of secrecy towards mental illness because of stigma (De Anstiss \& Ziaian, 2010; Michelson \& Sclare, 2009). Consequently, this unfamiliarity with talking as treatment results in the need to fully explain the processes and the benefits of the approach (Chase et al., 2008), such as common techniques, like the Subjective Units of Distress scales (Kiyimba and OReilly, in press).

Dynamics of therapy such as the need for an individual to reflect on their problems between sessions is essential to achieving better outcomes, but may not be understood by these young people. In addition, the need to address the trauma may also be part of therapy, but the young person may perceive this as unnecessary and painful, thus potentially damage the therapeutic relationship. The participants were avoidant of talking about past events, and of revisiting 
their memories of past adversities. Previous qualitative research showed similar findings, where young people talked about their main anxieties as linked to present circumstances, their asylum applications, loneliness and isolation (Brownlees \& Finch, 2010). A suggestion of talking therapy for them can be quite meaningless (Lynch, 2001). This, however, does not mean that their distress should not be acknowledged.

The findings from this study need to be contextualised in relation to its limitations as participants were from one area in England, and may not represent the refugee minors residing in other countries, and were mostly male. Additionally, while the young people’s language barrier posed some practical challenges in gathering and interpreting data from the interviews, in general the interviews were coherent and rich in qualitative information and much of the issues raised reflect the cultural and age-related issues that are likely across this population. The information generated in this project clearly establishes some of the barriers that these young people are experiencing and should not be dismissed.

In conclusion, the development of cost effective services may need to consider certain factors when designing care for these young people. This may require additional resources or time to explore the therapeutic relationship. Although it may be unrealistic finding a practitioner who is perfectly suited through culture or gender for a specific child, these factors will need consideration. Practitioners may already have some training to tackle this, but this should be considered a requirement for good practice. In developing services further, future research may inform care pathways through the triangulation of young people's views, with those of professionals, agencies, and commissioning bodies, especially in terms of their perceptions of talking treatments. Of importance is to examine the language barriers faced, and the passive 
acceptance of authority. This could be integrated with outcomes-focused longitudinal interventions. 


\section{References}

Bevin, T. (1999). Multiple traumas of refugees--near drowning and witnessing of maternal rape: Case of Sergio, age 9, and follow-up at age 16. Play therapy with children in crisis: Individual, group, and family treatment (2nd ed., pp. 164-182). New York: The Guildford Press.

Braun, V., \& Clarke, V. (2006). Using thematic analysis in psychology. Qualitative Research in Psychology, 3(2), 77-101.

Brownlees, L., \& Finch, N. (2010). Levelling the playing field; A UNICEF UK report into provision of services to unaccompanied or separated migrant children in three local authority areas in England UNICEF.

Chase, E., Knight, A., \& Statham, J. (2008). Promoting the emotional wellbeing and mental health of unaccompanied young people seeking asylum in the UK. London: Thomas Coram Research Unit, Institute of Education, University of London.

Chiumento, A., Nelki, J., Dutton, C., \& Hughes, G. (2011). School-based mental health service for refugee and asylum seeking children: Multi-agency working, lessons for good practice. Journal of Public Mental Health, 10(3), 164-177. 
Creed, T. A., \& Kendall, P. C. (2005) Therapist alliance-building behavior within a cognitive-behavioral treatment for anxiety in youth. Journal of Consulting and Clinical Psychology, 73, 498- 505.

De Anstiss, H., \& Ziaian, T. (2010). Mental health help-seeking and refugee adolescents: Qualitative findings from a mixed-methods investigation. Australian Psychologist, 45(1), 2937.

de Anstiss, H., Ziaian, T., Procter, N., Warland, J. \& Baghurst, P. (2009). Help-seeking for mental health problems in young refugees: a review of the literature with Implications for policy, practice, and research. Transcultural Psychiatry, 46, 584-607.

Derluyn, I., \& Broekaert, E. (2007). Different perspectives on emotional and behavioural problems in unaccompanied refugee adolescents. Ethnicity and Health, 12, 141-162.

DiGiuseppe, R., Linscott, J., \& Jilton, R. (1996) Developing the therapeutic alliance in childadolescent psychotherapy. Applied and Preventive Psychology, 5, 85- 100.

Ehnholt, K., \& Yule, W. (2006). Practitioner review: Assessment and treatment of refugee children and adolescents who have experienced war-related trauma. Journal of Child Psychology and Psychiatry, 47, 1197-1210.

Ellis, B. H., Miller, A., Baldwin, H., \& Abdi, S. (2011). New directions in refugee youth mental health services: Overcoming barriers to engagement. Journal of Child and Adolescent Trauma, 4, 69-85. 
Fazel, M., \& Stein, A. (2002). The mental health of refugee children. Archives of Disease in Childhood, 87(5), 366-370.

Fazel, M., Reed, R. V., Panter-Brick, C., \& Stein, A. (2012). Mental health of displaced and refugee children resettled in high-income countries: Risk and protective factors. The Lancet, 379, 266-282.

Fazel, M., Wheeler, J., \& Danesh, J. (2005). Prevalence of serious mental disorder in 7000 refugees resettled in western countries: A systematic review. The Lancet, 365, 1309-1314.

Francis, J. J., Johnston, M., Robertson, C., \& et al. (2010). What is an adequate sample size? operationalising data saturation for theory-based interview studies. Psychology and Health, 25(10), 1229-1245.

Griffith, P., \& Chan-Kam, S. (2002). Chapter 11: Why do we hate refugees? public opinion, citizenship and integration. In P. Griffith, \& M. Leonard (Eds.), Reclaiming Britishness (pp. 91-103). London: London Foreign Policy Centre (FPC).

Harris, E. M. (2008). Outreach as a component of mental health services for refugee children. Unpublished Doctor of Philosophy and Psychology, University of Illinois, Chicago.

Honwana, A. (2006). Chapter 13: Child soldiers: Community healing and rituals in Mozambique and Angola. International perspectives on youth conflict and development (pp. 225-244). New York: Oxford University Press. 
Huemer, J., \& Vostanis, P. (2010). Chapter 16: Child refugees and refugee families. In D. Bhugra, T. Craig \& K. Bhui (Eds.), Mental health of refugees and asylum seekers (pp. 225242). New York: Oxford University Press.

Kennedy, J., Seymour, D. J., \& Hummel, B. J. (1999). A comprehensive refugee health screening program. Public Health Reports, 114(5), 469-477.

Kia-Keating, M., \& Ellis, B. H. (2007). Belonging and connection to school in resettlement: Young refugees, school belonging, and psychosocial adjustment. Clinical Child Psychology and Psychiatry, 12(1), 29-43.

Kiyimba, N., and O’Reilly, M., (in press). The clinical use of Subjective Units of Distress scales (SUDs) in child mental health assessments: A thematic evaluation. Journal of Mental Health,

Legislation.gov.uk. (2010). Retrieved from http://www.legislation.gov.uk/ukpga/2010/15/contents

Lustig, S. L., Kia Keating, M., Knight, W. G., Geltman, P., Ellis, H., Kinzie, J. D., Keane, T.M., \& Saxe, G. N. (2004). Review of child and adolescent refugee mental health. Journal of the American Academy of Child and Adolescent Psychiatry, 43, 24-36. 
Lynch, M. A. (2001). Providing health care for refugee children and unaccompanied adolescents. Medicine, Conflict and Survival, 17, 125-130.

Majumder P. (2015). Service development in the new NHS. British Journal of Healthcare Management, 20(8), 224-229.

Mawn, L., Welsh, P., Stain, H., and Windbank, P. (2015). Youth speak: Increasing engagement of young people in mental health research. Journal of Mental Health, 24(5), 271275

Michelson, D., \& Sclare, I. (2009). Psychological needs, service utilization and provision of care in a specialist mental health clinic for young refugees: A comparative study. Clinical Child Psychology and Psychiatry, 14(2), 273-296.

O’Reilly, M., \& Parker, N. (2013). ‘Unsatisfactory Saturation’: A critical exploration of the notion of saturated sample sizes in qualitative research. Qualitative Research, 13, 190-197.

Priebe, S., \& McCabe, R. (2008). Therapeutic relationships in psychiatry: The basis of therapy or therapy in itself? International Review of Psychiatry, 20 (6), 521-526.

Rutter, J. (2003). Working with refugee children. York, UK: Joseph Rowntree Foundation.

Sanchez-Cao, E., Kramer, T., \& Hodes, M. (2012). Psychological distress and mental health service contact of unaccompanied asylum-seeking children. Child: Care, Health and Development, 39(5), 651-659. 
Söderbäck, M. (2011). The importance of including both a child perspective and the child’s perspective within health care settings to provide truly child-centred care. Journal of Child Health Care, 15 (2), 99-106.

Summerfield, D. (2000). Childhood, war, refugeedom and "trauma": Three core questions for mental health professionals. Transcultural Psychiatry, 37(3), 417-433.

Thabet, A., Karim, K., \& Vostanis, P. (2006). Trauma exposure in pre-school children in a war zone. British Journal of Psychiatry, 188, 154-158.

Thomas, L. K. (2007). The therapeutic needs of those fleeing persecution and violence, now and in the future. Resilience, suffering and creativity: The work of the refugee therapy centre (pp. 45-61). London: Karnac Books Ltd.

Thomas, S., \& Byford, S. (2003). Research with unaccompanied children seeking asylum. British Medical Journal, 327, 1400-1402.

United Nations High Commissioner for Refugees (UNHCR). (2012). Retrieved from http://www.unhcr.org/cgi-bin/texis/vtx/page?page=49e48f166\&submit=GO

Westermeyer, J. (1991). Psychiatric services for refugee children: An overview. In F. L. Ahearn, \& J. L. Athey (Eds.), Refugee children: Theory, research, and services (pp. 127162). Baltimore: Johns Hopkins University Press. 
